\title{
Automated Primary Particle Sizing of Nanoparticle Aggregates by TEM Image Analysis
}

\author{
Ramin Dastanpour ${ }^{1}$, Jocelyne M. Boone ${ }^{1}$ and Steven N. Rogak ${ }^{1 a}$, \\ ${ }^{1}$ Department of Mechanical Engineering, University of British Columbia \\ 6250 Applied Sciences Lane, Vancouver, BC, Canada, V6T $1 Z 4$ \\ ${ }^{a}$ Corresponding author: e-mail: rogak@mech.ubc.ca; Fax: +1-604-822-2403
}

\begin{abstract}
Nanoparticle aggregates formed in colloidal or aerosol processes have complex morphologies. Soot, for example, is formed as aggregates of primary particles. Soot properties are influenced by the diameter $\left(d_{\mathrm{p}}\right)$ and the arrangement of its constitutive primary particles. Transmission Electron Microscopy (TEM) is commonly used for the characterization of agglomerates' morphology, but manual analysis of the micrographs is extremely labour-intensive. Here, a new method is developed for automatic determination of the average primary particles diameter based on the variation of the 2-D pair correlation function $\mathrm{P}(r)$ at distances $r$ measured from the main skeleton of the aggregates. It is assumed that $\mathrm{P}^{*}=\mathrm{P}\left(d_{\mathrm{p}} / 2\right)$ is nearly constant. The method has been applied to numerical agglomerates and real soot aggregates collected from several operating conditions of a gasoline direct injection engine and a heavy-duty compressionignition engine. Using a constant value for $\mathrm{P}^{*}$ determined from the analysis of TEM images results in primary particle sizing errors (relative to manual sizing) of $\sim 13 \%$ for single aggregates. The ensemble-mean values of $d_{\mathrm{p}}$ for manual and automatic sizing differed by $\sim 4 \%$.
\end{abstract}

\section{Introduction}

Aerosol and colloidal aggregates are formed in many industrial and natural environments. These nanoparticle aggregates can be either useful or hazardous. Soot, for example, is formed by incomplete combustion of hydrocarbons; and has significant adverse impacts on human health [1] and climate [2,3]. The morphology of the aggregate influences its environmental impacts, and its transport and radiative properties; and primary particle diameter $\left(d_{\mathrm{p}}\right)$ is one of the most important morphological parameters of nano-clusters. 
Production rate and properties of synthetic particles, and accurate measurement of emission rate and size distribution of fractal aggregates depend on accurate estimation of the morphology of these particles and sizing of their constitutive monomers. Radiative properties of soot [4-8] and its impact on atmosphere [9] are non-linearly proportional to the diameters of its constitutive primary particles. For example, light scattering and absorption cross sections of soot are proportional to the $6^{\text {th }}$ and $3^{\text {rd }}$ powers of $d_{\mathrm{p}}$ [7], respectively. Soot toxicity $[10,11]$ and mass also correlate to the $2^{\text {nd }}$ and $3^{\text {rd }}$ powers of $d_{p}$, respectively. Advanced data inversion and accurate measurement of concentration and size distributions of soot particles using Scanning Mobility Particle Spectrometers (SMPS) [12] rely on accurate estimation of the primary particle diameter.

Combined light scattering and absorption measurement were used by Sorensen et al. [13] to estimate $d_{p}$. However, this approach requires prior information on the fractal dimension and optical properties, e.g. refractive index, of soot particles. Light depolarization has also been used for the measurement of $d_{\mathrm{p}}$; however, the method has been shown to be sensitive to the fractal dimension, refractive index, and a few other morphology parameters of soot [14]. Laser-Induced Incandescence (LII) is another method which uses cooling rate of the soot particles to measure primary particle size $[4,15]$. This model is also highly sensitive to soot chemical composition and morphology. Eggersdorfer et al. $[16,17]$ have proposed a method for sizing of the primary particles in Zirconium aggregates using particle mass and mobility diameter. This method is extended to carbon soot particles by Dastanpour et al. [18].

Transmission Electron Microscopy (TEM) of particles collected on a microscope grid provides visual observation of the soot particles and is commonly used for morphology characterization of nano particles [11,19-27]. Statistically reliable results can only be acquired from this method when a great number of images are analysed. Primary particle diameter are commonly measured manually [20,28] from digitized TEM images; and this manual sizing is an exceedingly time-intensive process.

Grishin et al. [29] have used Hough transform in the analysis of the TEM images for automatic determination of primary particle size distribution in individual aggregates. However, accurate results can only be acquired when prior information on the size range of the primary particles in each aggregate is known. This model can only detect primary particles touching the edge of the aggregate and relies on the assumption of perfectly spherical structures for primary particles. Testing this method on samples from a Gasoline Direct Injection (GDI) engine (described below), we found that the accuracy of this model is extremely sensitive to the fitting parameters. For example, by changing the engine operating condition an increase of approximately $4 \mathrm{~nm}$ in primary particle diameter was measured from manual analysis of the TEM images; however the automatic method only showed an increase of less than $1 \mathrm{~nm}$ in primary particle size. Accurate measurement of the primary particle diameter by this method requires algorithm calibration for each individual TEM image. 
In another automated sizing algorithm [30], the Euclidian Distance Mapping SurfaceBased Scale Analysis (EDM-SBS) was used for automatic sizing of the primary particles. However the influence of particle size on the accuracy of the method has not been investigated. De Temmerman et al. [31] have developed a semi-automatic image processing method in which the primary particles are detected on binarized TEM images by watershed segmentation and their sizes are measured using EDM. Their method is not limited by the assumption of spherical structures for primary particles and monomers should not necessarily be located on the perimeter of the clusters.

A simple and robust method for automatic determination of average diameter of the primary particles in individual aggregates has been developed here and then tested on numerical agglomerates of point touching primary particles and soot aggregates formed at different operating conditions of two reciprocating engines. This method can be used as an alternative approach for automatic measurement of the primary particle diameters in individual $^{2}$ and ensembles ${ }^{3}$ of agglomerates.

\section{The Pair Correlation Method (PCM)}

This method uses the 2-D pair correlation function to estimate $d_{p}$. The pair correlation function $\mathrm{P}(r)$ is a weighted average of the particle density at a given radius $r$ [32]. For binary images, this function estimates the probability of finding another pixel at a distance $r$ from a reference pixel:

$\mathrm{P}(r)=\lim _{d r \rightarrow 0} \frac{1}{N} \frac{\sum_{\mathrm{i}=1}^{N} \sum_{\mathrm{j}=1}^{N} \theta\left(r_{\mathrm{ij}}\right)}{2 \pi r d r}$

where $\theta\left(r_{\mathrm{ij}}\right)=1$ for $r<r_{\mathrm{ij}}<r+d r$ and $\theta\left(r_{\mathrm{ij}}\right)=0$ elsewhere, $r_{\mathrm{ij}}$ is the distance between pixels $\mathrm{i}$ and $\mathrm{j}$, and $N$ is total number of the pixels within the projected region of the particle. At small distances, $\mathrm{P}(r) \rightarrow 1$; at distances comparable to the aggregate dimensions it falls to zero.

Simple Pair Correlation Method (PCM) developed here is based on the assumption that $\mathrm{P}^{*}=\mathrm{P}\left(d_{p} / 2\right)$ is nearly constant for all soot particles. First, the value of $\mathrm{P}^{*}$ is measured for aggregates with known primary particle diameter. To this end, synthetic TEM images were produced by random projections of straight chains of monodisperse primary particles and numerical fractal agglomerates with different levels of primary particle polydispersity and overlap. This parameter was also measured for real TEM images using manually measured primary particle radius. $\mathrm{P}(r)$ was calculated for all synthetic and real TEM images, and its value at the known primary particle radius $\left(\mathrm{P}^{*}\right)$ was measured. Finally, a constant value was selected for $\mathrm{P}^{*}$ by averaging $\mathrm{P}\left(d_{p} / 2\right)$ measured for individual synthetic and real TEM images.

\footnotetext{
${ }^{2}$ Average primary particle diameter for each aggregate

${ }^{3}$ Average primary particle diameter in the whole ensemble of aggregates. This is the average of the mean primary particles measured for each individual aggregate.
} 
Once $\mathrm{P}^{*}$ is known, PCM can be used for the calculation the average diameter of the primary particles in both synthetic and real TEM images. This method consists of three main steps. First, the portion of the image corresponding to the projection of each individual aggregate should be detected. To this end, real TEM images were automatically binarized using Otsu's algorithm for thresholding [33] and rolling ball transformation [29]; and synthetic images were scanned pixel-by-pixel. Second, $\mathrm{P}(r)$ should be calculated for each aggregate. In order to reduce computational costs, but still preserve the structure of the aggregates, values of the pair correlation function (PCF) were computed at different distances $r$ from the reference points on the skeleton of the particles. The particle's skeleton was detected using MATLAB built-in function "bwmorph" and operation algorithm "thin". This operation removes pixels on the boundaries of the projected binary image without breaking the particle. Skeleton and $\mathrm{P}(r)$ curve of a sample TEM image are illustrated in Figure 1. Finally, average radius of the primary particles will be the radius at which $\mathrm{P}(r)$ is equal to $\mathrm{P}^{*}$ (Fig. 1, panel d).

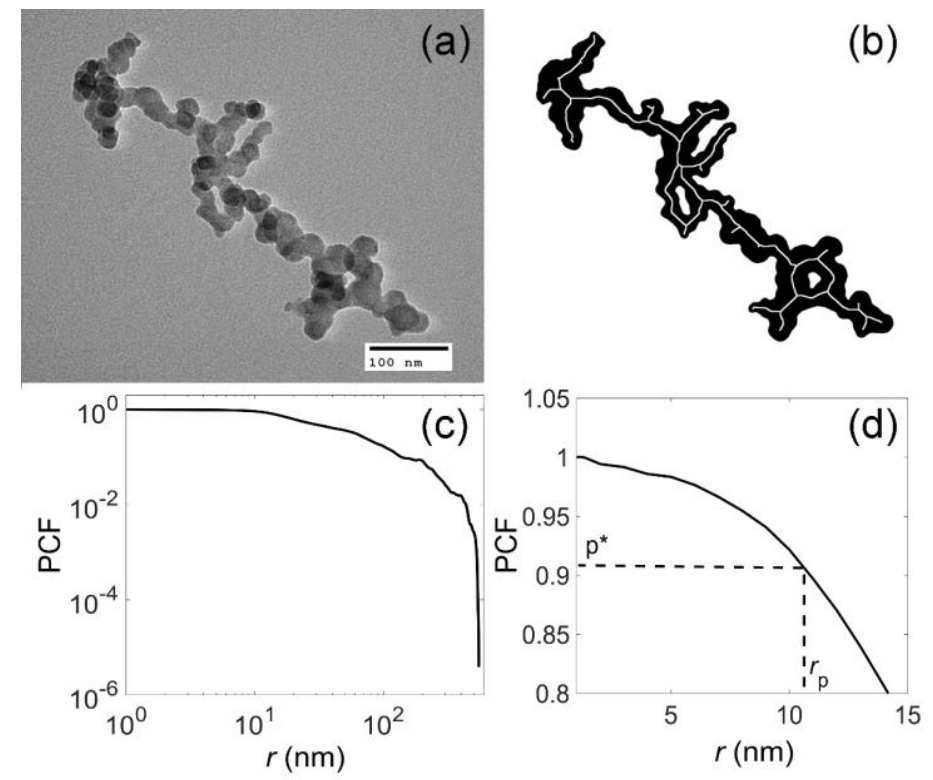

Figure 1: (a) Sample TEM image of real soot; (b) its skeleton; (c) $\mathrm{P}(r)$; (d) detail of $\mathrm{P}(r)$ around $\mathrm{P}^{*}$ and $r_{\mathrm{p}}$.

\section{Images used for PCM development and validation}

\subsection{Numerical agglomerates}

The PCM was first developed using numerical particles including straight chains and three-dimensional fractal agglomerates. Straight chains were composed of monodisperse primary particles of point-touching monomers (overlap coefficient ${ }^{4} C_{\mathrm{ov}}=0$ ). The total number of the monomers $\left(N_{\mathrm{p}}\right)$ in chains was varied between 1 and 100 . Fractal

${ }^{4} C_{\mathrm{ov}}=\left(\overline{d_{\mathrm{p}, l]}}-D_{i j}\right) / \overline{d_{\mathrm{p}, l j}}$, where $\overline{d_{\mathrm{p}, l j}}$ and $D_{i j}$ are the average diameter and center-to-center separation distance of two adjustment monomers of $i$ and $j$. 
agglomerates were generated by Eggersdorfer and Pratsinis [34]. The agglomerates were produced by a cluster-cluster agglomeration algorithm [34,35] and consisted of 16, 32, $64,128,256$, and 512 primary particles. Fractal prefactor, $k_{\mathrm{f}}$, of the generated particles was approximately constant and equal to 1.4 while the fractal dimension, $D_{\mathrm{f}}$, decreased from 1.78 to 1.73 as the primary particles polydispersity increased from 1 to 1.6, consistent with the results obtained by Eggersdorfer \& Pratsinis [36] for particles generated by the DLCA algorithm. Diameters of the primary particles were selected randomly from log-normal distributions with geometric standard deviations $\left(\sigma_{\mathrm{g}}\right)$ of 1.0, $1.2,1.4$, and 1.6. Considering the range of the values reported for the overlap coefficient of soot in literature [19,37,38], three different cases of $C_{\mathrm{ov}}=0$ (point-touching monomers), $C_{\mathrm{ov}}=0.1$, and $C_{\mathrm{ov}}=0.3$ were considered for each $N_{\mathrm{p}}$ and $\sigma_{\mathrm{g}}$. The range of the parameters considered here covers a large group of fractal structures ranging from gold nanochains to zirconia and soot aggregates. Ten aggregates were generated for each $N_{\mathrm{p}}, \sigma_{\mathrm{g}}$, and $C_{\mathrm{ov}}$, and 50 random synthetic TEM images were produced for each aggregate, a total of 36,000 synthetic images. A sample numerical agglomerate and the corresponding synthetic TEM image is illustrated in Figure 2.

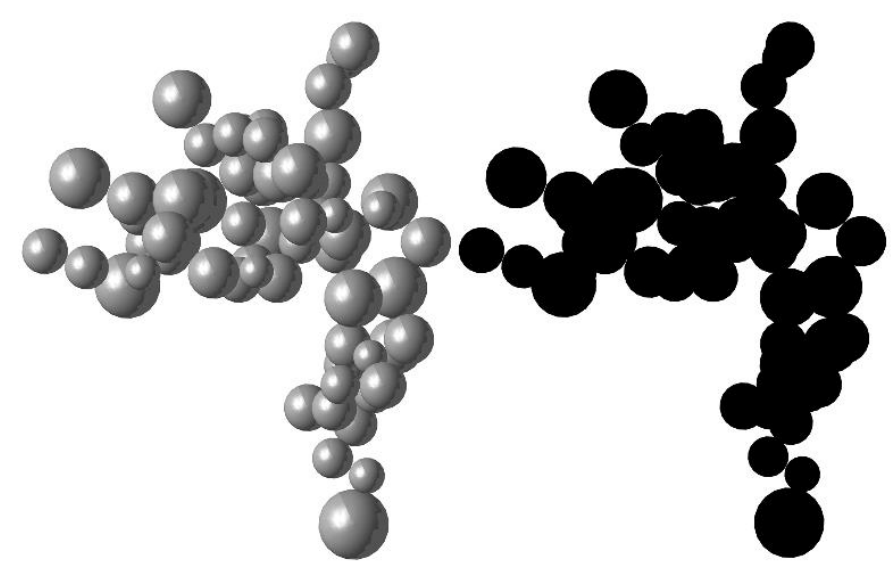

Figure 2: Sample numerical agglomerate (left) and its synthetic TEM image (right).

\subsection{Images of engine exhaust particulate}

Soot particles were collected from several operating conditions of two reciprocating engines. A Ford 2.0 Liter, inline 4-cylinder GDI engine was run with three types of fuel (gasoline blended with 0\%, 10\%, and 30\% ethanol: E0, E10, and E30, respectively). Operating conditions of this engine include transient cold start (CS) and hot start (HS) tests, simulated highway cruise (C) condition (2600 RPM and 58 N.m torque), and a higher speed and lower torque (S) setting (3000 RPM and 38 N.m torque). Another set of samples were collected from a 15 liter, 6-cylinder (5 cylinders were deactivated), Cummins ISX High Pressure Direct Injection (HPDI) engine operating with Westport Innovations HPDI natural gas combustion system. This engine was run at five different operating modes including $25,37,50$, and 75 percent of maximum load at an engine speed of $1500 \mathrm{rpm}$ (B25, B37, B50, and B75). Lower load test points were operated at 
$20 \%$ exhaust gas recirculation (EGR) while the highest load (B75) test point was ran at $25 \%$ EGR. Engine was also run at 63\% maximum load and a lower speed of 1200 RPM at $0 \%$ EGR (A63). Soot samples were deposited thermophoretically on TEM grids and images were produced by a Hitachi $\mathrm{H} 7600$ transmission electron microscope operating at $80 \mathrm{kV}$ under high resolution mode. Details of the experiments and sampling procedures are further discussed in $[18,20,26]$. Several studies have shown that soot from combustion sources, unless it has been collapsed by substantial coatings (not the case here) has a fractal dimension, $D_{\mathrm{f}}$, close to $1.8[39,40]$. Soewono studied the fractal dimension from one of the test engines here (the HPDI engine) and found only subtle variations in $D_{\mathrm{f}}[41,42]$.

\section{Results and discussion}

\subsection{Numerical aggregates}

Each of the synthetic chains and fractal agglomerates were projected randomly into 50 different orientations ${ }^{5}$ (500 images for each set of $N_{\mathrm{p}}, \sigma_{\mathrm{g}}$, and $C_{\mathrm{ov}}$ for fractal aggregates and 50 images for each chain size). Synthetic binary images were produced by Monte Carlo approach: each particle was scanned by rays in $\mathrm{Z}$ direction emerging from every single point on the X-Y plane. Once the binary images were produced, skeleton of the projected particle was detected and pair correlation function was computed at distances $r$ from the skeleton of each image using Eq. (1); and values of $\mathrm{P}^{*}$ were calculated at the known average $r_{\mathrm{p}}$.

Variation of the average $\mathrm{P}^{*}$ with the particle size $\left(N_{\mathrm{p}}\right)$ is illustrated in Figure 3 (left panel) for chains and fractal agglomerates of point-touching monomers $\left(C_{\mathrm{ov}}=0\right)$. The effect of the overlap coefficient on $\mathrm{P}^{*}$ of the fractal aggregates is reported in Table 1 and illustrated in Figure 4. In Figure 4, the horizontal axis is the ratio of the area-equivalent and gyration diameters $d_{\mathrm{a}} / d_{\mathrm{g}}$. This ratio decreases with $N_{\mathrm{p}}$ for fractal dimension <2, and unlike the fractal dimension, is obtained directly from the image without assumptions.

As illustrated in Figures 3 (left panel) and $4, \mathrm{P}^{*}$ increases with $N_{\mathrm{p}}, \sigma_{\mathrm{g}}$ and $C_{\mathrm{ov}}$. As particles become larger, a greater portion of the primary particles are shielded by other monomers which ultimately results in slower decrease of the value of the pair correlation function with $r$ and a larger value for $\mathrm{P}^{*}$. As $C_{\mathrm{ov}}$ increases, the average thickness (distance between the skeleton and the edge) of the projected particle also increases; which ultimately results in an increase in the value of $\mathrm{P}^{*}$.

As $N_{\mathrm{p}}$ increases, $\mathrm{P}^{*}$ approaches $0.7\left(N_{\mathrm{p}} \leq 100\right)$ for chains of monodisperse primary particles. Average $\mathrm{P}^{*}$ varies between 0.84 to 0.95 for aggregates of different $N_{\mathrm{p}}, \sigma_{\mathrm{g}}$ and

\footnotetext{
${ }^{5}$ To insure the accuracy of the algorithm, ten particles were selected and projected randomly into 50 different orientations. Measured average $\mathrm{P}^{*}$ was consistent $(<0.01$ deviation) with those obtained from 20 projections.
} 
$C_{\mathrm{ov}}$. Its average varies between 0.89 and 0.93 for aggregates of $\sigma_{\mathrm{g}} \cong 1.2$ and $C_{\mathrm{ov}}$ in the range of 0.1 and 0.3 ; which are the most probable primary particle polydispersity (Fig.3 of [20]) and overlap coefficients [19,37,38] for real soot aggregates. Compared to chains, fractal agglomerates have a branched structure and more particle overlap in projected images, which might explain the larger $\mathrm{P}^{*}$.
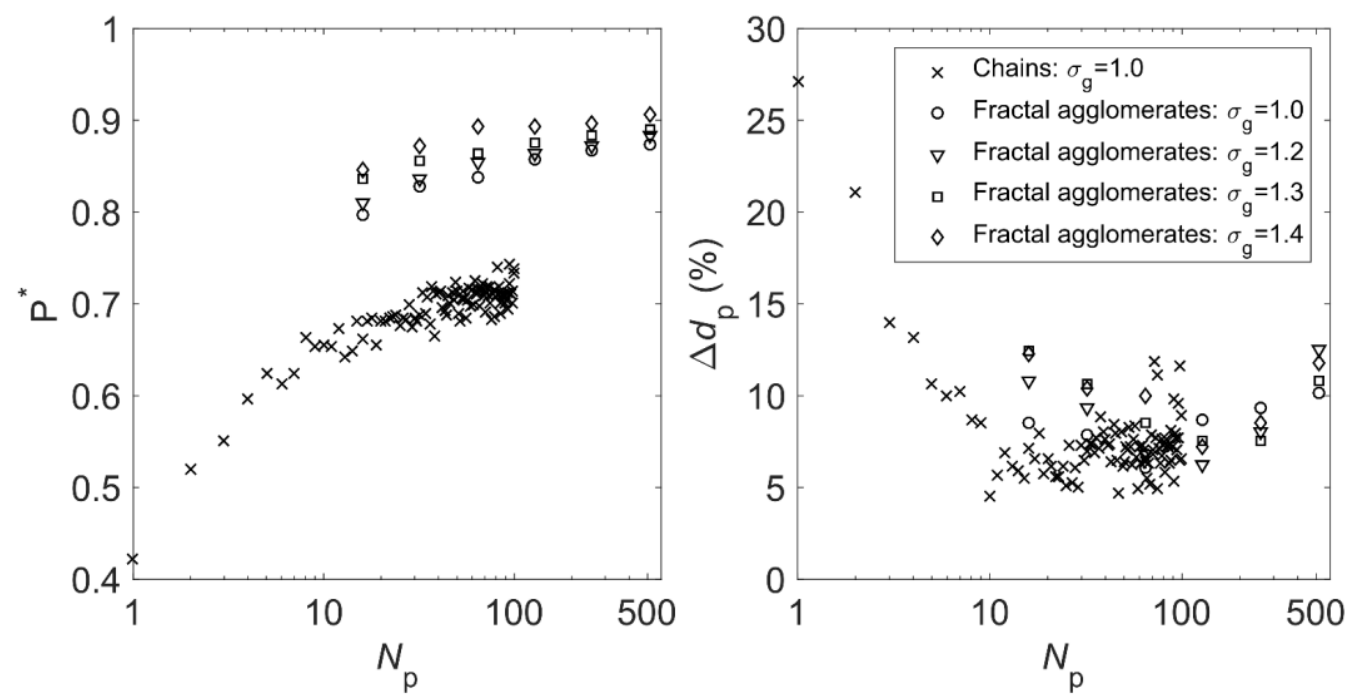

Figure 3: $\mathrm{P}^{*}$ of straight chains and agglomerates of point-touching primary particles with different sizes and polydispersity (left panel); measurement error of PCM. Each point represents the average of the desired parameter over 50 synthetic images for chains and 500 synthetic images for agglomerates.

Table 1: $\mathrm{P}^{*}$ for agglomerates of different $N_{\mathrm{p}}$ and $\sigma_{\mathrm{g}}{ }^{1}$

\begin{tabular}{|c|c|c|c|c|c|c|c|c|}
\hline$\sigma_{\mathrm{g}}$ & $C_{\mathrm{v}}$ & $N_{\mathrm{p}}=16$ & $N_{\mathrm{p}}=32$ & $N_{\mathrm{p}}=64$ & $N_{\mathrm{p}}=128$ & $N_{\mathrm{p}=256}$ & $N_{\mathrm{p}=512}$ & Average \\
\hline \multirow{4}{*}{1.0} & 0.0 & 0.80 & 0.83 & 0.84 & 0.86 & 0.87 & 0.87 & 0.84 \\
\cline { 2 - 9 } & 0.1 & 0.84 & 0.87 & 0.88 & 0.89 & 0.90 & 0.91 & 0.88 \\
\cline { 2 - 9 } & 0.3 & 0.89 & 0.92 & 0.93 & 0.94 & 0.94 & 0.94 & 0.93 \\
\hline \multirow{4}{*}{1.2} & 0.0 & 0.81 & 0.84 & 0.85 & 0.86 & 0.87 & 0.88 & 0.85 \\
\cline { 2 - 9 } & 0.1 & 0.85 & 0.88 & 0.89 & 0.90 & 0.91 & 0.92 & 0.89 \\
\cline { 2 - 9 } & 0.3 & 0.90 & 0.93 & 0.93 & 0.94 & 0.95 & 0.95 & 0.93 \\
\hline \multirow{4}{*}{1.4} & 0.0 & 0.84 & 0.86 & 0.86 & 0.87 & 0.88 & 0.89 & 0.87 \\
\cline { 2 - 9 } & 0.1 & 0.85 & 0.89 & 0.90 & 0.91 & 0.91 & 0.92 & 0.90 \\
\cline { 2 - 9 } & 0.3 & 0.92 & 0.94 & 0.94 & 0.95 & 0.95 & 0.96 & 0.94 \\
\hline 1.6 & 0.0 & 0.85 & 0.87 & 0.89 & 0.89 & 0.90 & 0.91 & 0.88 \\
\cline { 2 - 8 } & 0.1 & 0.88 & 0.91 & 0.92 & 0.93 & 0.93 & 0.93 & 0.92 \\
\cline { 2 - 9 } & 0.3 & 0.93 & 0.95 & 0.96 & 0.96 & 0.96 & 0.97 & 0.95 \\
\hline
\end{tabular}

${ }^{1}$ standard deviation of the reported $\mathrm{P}^{*}$ varies between 0.01 and 0.05 for large and small $N_{\mathrm{p}}$, respectively. 

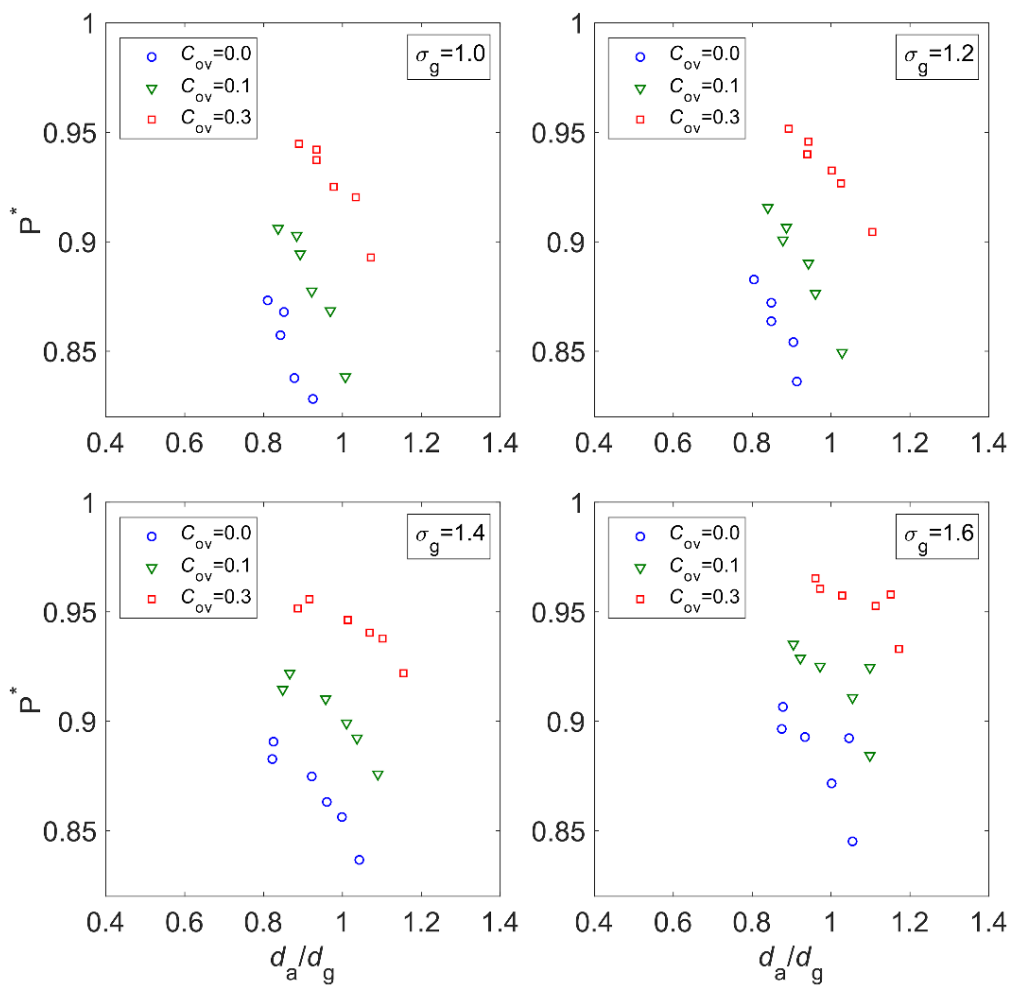

Figure 4: Effect of on $C_{\mathrm{ov}}$ on $\mathrm{P}^{*}$ of aggregates of different sizes and polydispersity.

As reported by Dastanpour and Rogak [43], the gyration diameter of the fractal agglomerates increases with the polydispersity of the primary particles. Accordingly, $\mathrm{P}^{*}$ is also expected to increase with $\sigma_{\mathrm{g}}$, but results here show the effect is small for large aggregates. The effect of $\sigma_{\mathrm{g}}$ on $\mathrm{P}^{*}$ can be estimated for numerical agglomerates of pointtouching monomers using the following correlation $\left(\mathrm{R}^{2}=0.99\right)$ :

$\mathrm{P}_{\mathrm{avg}}^{*}=0.78+0.022 \exp \left(\sigma_{\mathrm{g}}\right)$

Next, the constant values of $\mathrm{P}^{*}$ obtained for chains (0.7), and fractal agglomerates of different polydispersity (right column of Table 1) were used to estimate $d_{\mathrm{p}}$ from the pair correlation functions, $\mathrm{P}(r)$, calculated for each synthetic image. The deviation of the estimated diameter $d_{\mathrm{p}, \mathrm{PCM}}$ from the real average primary particle diameter $d_{\mathrm{p} \text {,real }}$ was estimated using Eq. (3) and is illustrated in Figure 3 (right panel). For simplicity, only results obtained for $C_{\mathrm{ov}}=0$ are illustrated.

$\Delta d_{\mathrm{p}}=100 \cdot\left|\frac{d_{\mathrm{p}, \mathrm{PCM}}-d_{\mathrm{p}, \text { real }}}{d_{\mathrm{p}, \text { real }}}\right|$

This method resulted in an average primary particle sizing error of approximately $7 \%$ for straight chains of $N_{\mathrm{p}}>10$ and less than $14 \%$ error for fractal agglomerates of different sizes and polydispersity. Maximum error occurs for single spheres and is approximately 
$27 \%$. If the aggregated-optimized value of $\mathrm{P}^{*}$ is used for analysis of straight chains, the error is approximately $18 \%$ (Fig. SI1, online supplemental information).

\subsection{Real soot aggregates}

First, primary particle size was measured manually for 11 operating conditions of two reciprocating engines. On average 35 aggregates were analyzed for each operating condition. Then, Otsu's algorithm and rolling ball methods were used for automatic detection of the aggregate projected area; and the corresponding pair correlation functions $\mathrm{P}(r)$ were calculated. Using $\mathrm{P}^{*}=0.85$ obtained from the simulation of the numerical agglomerates of point-touching monomers with $\sigma_{\mathrm{g}} \cong 1.2$ (approximate polydispersity for real soot aggregates according to Fig. 3 in [20]), the average diameter of the primary particles was measured for individual aggregates. As shown in Figure SI2 (online supplemental information) the average sizing error of the model using $\mathrm{P}^{*}=0.85$ is approximately $20 \%$. Real aggregates often have partially fused primary particles, and primary particle overlap coefficients in the range of $0-0.3$ which suggest that the optimum $\mathrm{P}^{*}$ for soot should be larger than 0.85 , which indeed was found to be the case.

To further improve the accuracy of the method for carbon soot, $\mathrm{P}^{*}$ was measured for 132 aggregates randomly selected from the library of the TEM images taken at 5 different operating conditions of the GDI engine. Since the number of the primary particles cannot be directly measured from 2-D TEM images, $\mathrm{P}^{*}$ is plotted as a function of $d_{\mathrm{a}} / d_{\mathrm{g}}$ in Figure 5, as in Figure 4. The average $\mathrm{P}^{*}$ obtained from the analysis of the TEM images of the GDI engine is $0.913 \pm 0.02$. This is within the range of the $P^{*}$ values determined for synthetic aggregates with $1.2 \leq \sigma_{\mathrm{g}} \leq 1.4$ and $0.1 \leq C_{\mathrm{ov}} \leq 0.3$.

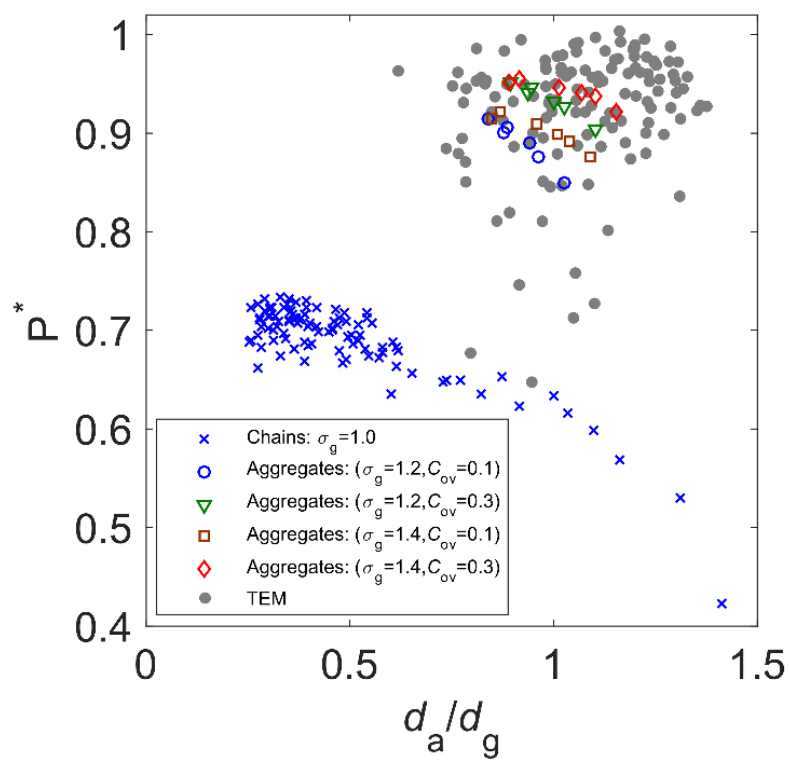

Figure 5: $\mathrm{P}^{*}$ of real soot aggregates ("TEM") as well as synthetic images of chains and fractal agglomerates. 
Using $\mathrm{P}^{*}=0.913$ obtained from the analysis of the TEM images produced for the GDI engine, average primary particle size was measured for 390 aggregates captured on TEM images of both GDI and HPDI engines. As summarized in Table 2 and Figure 6, the size of the primary particles measured manually varies between approximately $10 \mathrm{~nm}$ and 50 $\mathrm{nm}$ for individual particles; and its ensemble-average varies between $15 \mathrm{~nm}$ and $26 \mathrm{~nm}$ in different operating conditions. This shows that the proposed method is not limited to a narrow range of the primary particle size. PCM estimates the average diameter of the primary particles in individual aggregates $\left(d_{\mathrm{p}, \mathrm{agg}}\right)$ with an average error of approximately $13 \%$ for all operating conditions. Considering the nature of the manual sizing which is highly time-intensive, PCM provides results with useful level of accuracy in a very short processing time (less than 5 seconds for binarization and primary particle sizing on a Core(TM) i7-3770 CPU @ $3.40 \mathrm{GHz}, 8$ GB RAM). The ensemble average diameter of the primary particles can be estimated for each operating condition $\left(d_{\mathrm{p}, \text { ens }}\right)$ with an average error of $4 \%$.

Table 2: Average primary particle diameters obtained from manual image processing and PCM

\begin{tabular}{lcccc} 
& \multicolumn{2}{c}{ Diameters $(\mathrm{nm})$} & \multicolumn{2}{c}{ Errors $(\%)$} \\
\hline Operating condition & Manual & PCM & $\Delta d_{\mathrm{p}, \mathrm{ens}}{ }^{3}$ & $\Delta d_{\mathrm{p}, \mathrm{agg}}{ }^{4}$ \\
& $d_{\mathrm{p}, \mathrm{ens}, \mathrm{manual}}$ & $d_{\mathrm{p}, \mathrm{ens}, \mathrm{PCM}}$ & & \\
\hline GDI_E0_C & $26( \pm 7)^{2}$ & $24( \pm 9)$ & $7 \%$ & $17 \%$ \\
GDI_E0_S & $26( \pm 9)$ & $24( \pm 7)$ & $6 \%$ & $11 \%$ \\
GDI_E0_CS & $12( \pm 4)$ & $11( \pm 2)$ & $10 \%$ & $15 \%$ \\
GDI_E10_C & $21( \pm 6)$ & $22( \pm 7)$ & $3 \%$ & $10 \%$ \\
GDI_E10_S & $16( \pm 4)$ & $15( \pm 5)$ & $4 \%$ & $9 \%$ \\
GDI_E10_HS & $15( \pm 4)$ & $16( \pm 6)$ & $5 \%$ & $16 \%$ \\
GDI_E30_C & $17( \pm 4)$ & $16( \pm 4)$ & $5 \%$ & $11 \%$ \\
GDI_E30_S & $18( \pm 4)$ & $18( \pm 5)$ & $3 \%$ & $10 \%$ \\
GDI_E30_CS & $21( \pm 8)$ & $21( \pm 9)$ & $2 \%$ & $12 \%$ \\
HPDI_B50 & $19( \pm 5)$ & $18( \pm 6)$ & $3 \%$ & $13 \%$ \\
HPDI_B25 & $20( \pm 8)$ & $19( \pm 9)$ & $4 \%$ & $14 \%$ \\
HPDI_A63 & $26( \pm 7)$ & $21( \pm 6)$ & $3 \%$ & $11 \%$ \\
HPDI_B37 & $17( \pm 4)$ & $17( \pm 5)$ & $5 \%$ & $16 \%$ \\
HPDI_B75 & $17( \pm 4)$ & $17( \pm 5)$ & $2 \%$ & $13 \%$ \\
\hline
\end{tabular}

${ }^{1} d_{\mathrm{p} \text {,ens }}$ is the average diameter of the primary particles in the ensemble of images produced for each operating condition.

${ }^{2}$ Values in parenthesis are standard deviations.

${ }^{3} \Delta d_{\mathrm{p}, \mathrm{ens}}$ is the average error of the automatic method for ensembles of aggregates; and is equal to $100 \cdot\left(d_{\mathrm{p}, \text { ens,model }}-d_{\mathrm{p} \text {,ens,manual }}\right) / d_{\mathrm{p} \text {,ens,manual }}$.

${ }^{4} \Delta d_{\mathrm{p}, \text { agg }}$ is the average error for individual aggregates $=\frac{100}{N_{\mathrm{agg}}} \sum_{i}^{N_{\mathrm{agg}}}\left|\frac{d_{\mathrm{p}, \text { model, } \mathrm{i}}-d_{\mathrm{p}, \mathrm{TEM}, \mathrm{i}}}{d_{\mathrm{p}, \mathrm{TEM}, \mathrm{i}}}\right|$, where $N_{\text {agg }}$ is the total number of the aggregates analysed at each operating condition. 


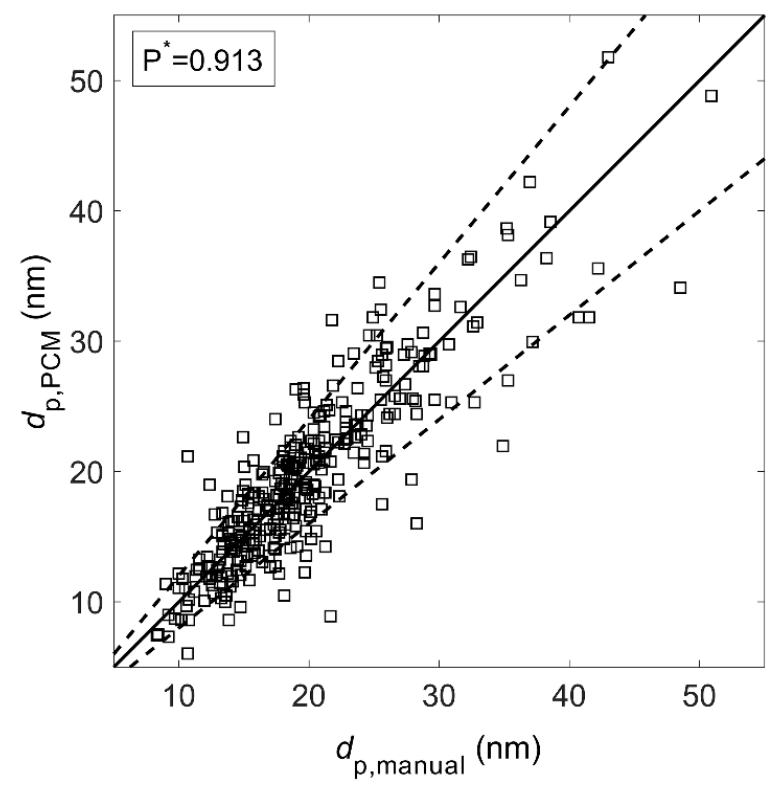

Figure 6: Average $d_{\mathrm{p}}$ for 390 individual aggregates by PCM vs. manual sizing ("TEM"). Solid line is a 1by- 1 line, and dashed lines are $\pm 20 \%$ error boundaries.

The accuracy of the PCM is sensitive to the $\mathrm{P}^{*}$ value; decreasing $\mathrm{P}^{*}$ from 0.913 to 0.82 , $\Delta d_{\text {p,ens }}$ increases to from $4 \%$ to $10 \%$. However, as described earlier, the optimum value of $\mathrm{P}^{*}=0.913$ is determined here by extensive analysis of thousands of synthetic and real TEM images and is shown to provide good accuracy for simulated and real soot particles.

The model accuracy was estimated using Eq.(3) assuming that the manual measurements were perfect. In reality, manual measurements are influenced by human perception and also involve statistical uncertainty, so not all of the reported error is attributable to flaws in the PCM. As an illustrative calculation, suppose that 16 primaries are measured manually for a large aggregate with $N_{\mathrm{p}} \gg 16$. The standard deviation is typically $8 \mathrm{~nm}$ (Table 2), yielding a standard error of $8 / \sqrt{16}=2 \mathrm{~nm}$ on a typical mean diameter of $20 \mathrm{~nm}$ (10\% error). Thus, roughly half of the reported error could be related the statistics of the manual sizing.

An attempt was made to correlate $\mathrm{P}^{*}$ with features that can be obtained directly from the image, such as $d_{\mathrm{a}} / d_{\mathrm{g}}$ which were shown earlier to affect $\mathrm{P}^{*}$. As shown in Figures SI3 and SI4, a simple correlation between the parameters described above and the value of the $\mathrm{P}^{*}$ cannot be easily derived. Although this "generalized" method can handle a wide range of particle types (eg. chains to aggregates) better than the simple PCM with constant $\mathrm{P}^{*}$, for normal soot the more complex method provides no improvement. A possible explanation is that one of the most important structural features, the overlap coefficient, is not easily extracted from the image.

When either versions of the PCM are used, errors associated with the human biases and manual analysis of the TEM images are eliminated. As illustrated in Figure SI6 (online supplemental Information), both versions of this method are capable of capturing a size 
correlation between the primary particle and aggregate sizes, consistent with the trends reported by Dastanpour and Rogak [20] from the manual analysis.

Primary particle overlap coefficient can also be measured by the PCM approach. If primary particle size is known from other methods (e.g. manual measurement or combined mass-mobility experiments as discussed in [18]), the value of $\mathrm{P}^{*}$ corresponding to the known monomer diameter can be calculated. Overlap coefficient can be estimated using $\mathrm{P}^{*}$ and $d_{\mathrm{a}} / d_{\mathrm{g}}$ (Figure 4 ).

To facilitate the application of this method by interested researchers, developed open source program is uploaded online and can be downloaded for free [44].

\section{Conclusions}

A new method, the Pair Correlation Method (PCM), was developed for automatic estimation of the average primary particle size from binary images of aggregates. The key assumption is that the pair correlation function drops to a critical value $\mathrm{P}^{*}$ at the primary particle size. Unfortunately $\mathrm{P}^{*}$ is not the same for all classes of aggregates, and is not a simple function of geometric parameters such as aspect ratio or the number of primary particles. Fortunately, for broad classes of particles, such as engine soot, $\mathrm{P}^{*}$ is nearly constant.

Although this method does not provide information on the size distribution of the primary particles in individual aggregates, it provides useful results for real soot. Ensembleaverage size distribution of the monomers can also be calculated by this method. $\mathrm{P}^{*}$ was tuned for a portion of the images from one engine and then tested on soot images from two types of reciprocating engines. Using a constant value of $\mathrm{P}^{*}$ resulted in an average error below $13 \%$ (relative to manual sizing) for individual aggregates and $4 \%$ for ensemble of the aggregates. This accuracy is comparable to the best available algorithms for primary particle sizing, and importantly, the PCM can be used for analysis of TEM images of agglomerates without a priori knowledge of the primary particle size.

\section{Acknowledgments}

M. Eggersdorfer and S. Pratsinis generously provided the numerically generated aggregates for the production of synthetic TEM images. The authors would also like to thank Jason Olfert, James Wallace, Brian Graves, Manuel Ramos, Phillip Mireault and Bronson Patychuk for their contributions to the sampling campaigns for GDI and HPDI engines. The authors also thank CREATE-AAP program at UBC for their financial support.

\section{References}

[1] M. Franchini, P.M. Mannucci, Air pollution and cardiovascular disease., Thromb. Res. 129 (2012) 230-4. doi:10.1016/j.thromres.2011.10.030. 
[2] J. Hansen, L. Nazarenko, Soot climate forcing via snow and ice albedos., Proc. Natl. Acad. Sci. U. S. A. 101 (2004) 423-8. doi:10.1073/pnas.2237157100.

[3] M.Z. Jacobson, Short-term effects of controlling fossil-fuel soot, biofuel soot and gases, and methane on climate, Arctic ice, and air pollution health, Geophys. Res. 115 (2010). doi:10.1029/2009JD013795.

[4] F. Liu, M. Yang, F. a. Hill, D.R. Snelling, G.J. Smallwood, Influence of polydisperse distributions of both primary particle and aggregate size on soot temperature in low-fluence LII, Appl. Phys. B. 83 (2006) 383-395. doi:10.1007/s00340-006-2196-z.

[5] P. Meakin, Formation of fractal clusters and networks by irreversible diffusion-limited aggregation, Phys. Rev. Lett. 51 (1983) 1119-1122. doi:10.1103/PhysRevLett.51.1119.

[6] R.D. Mountain, G.W. Mulholland, H. Baum, Simulation of aerosol agglomeration in the free molecular and continuum flow regimes, J. Colloid Interface Sci. 114 (1986) 67-81. doi:10.1016/0021-9797(86)90241-9.

[7] C.M. Sorensen, Light scattering by fractal aggregates: a review, Aerosol Sci. Technol. 35 (2001) 648-687. doi:10.1080/02786820117868.

[8] Q. Zhang, P. a. Rubini, Modelling of light extinction by soot particles, Fire Saf. J. 46 (2011) 96-103. doi:10.1016/j.firesaf.2010.11.002.

[9] K.S. Johnson, B. Zuberi, L.T. Molina, M.J. Molina, M.J. Iedema, J.P. Cowin, et al., Processing of soot in an urban environment: case study from the Mexico City Metropolitan Area, Atmos. Chem. Phys. 5 (2005) 3033-3043. doi:10.5194/acp-5-3033-2005.

[10] A.D. Maynard, E.D. Kuempel, Airborne Nanostructured Particles and Occupational Health, J. Nanoparticle Res. 7 (2005) 587-614. doi:10.1007/s11051-005-6770-9.

[11] S. Bau, O. Witschger, F. Gensdarmes, O. Rastoix, D. Thomas, A TEM-based method as an alternative to the BET method for measuring off-line the specific surface area of nanoaerosols, Powder Technol. 200 (2010) 190-201. doi:10.1016/j.powtec.2010.02.023.

[12] A.A. Lall, S.K. Friedlander, On-line measurement of ultrafine aggregate surface area and volume distributions by electrical mobility analysis: I. Theoretical analysis, J. Aerosol Sci. 37 (2006) 260-271. doi:10.1016/j.jaerosci.2005.05.021.

[13] C.M. Sorensen, J. Cai, N. Lu, Light-scattering measurements of monomer size, monomers per aggregate, and fractal dimension for soot aggregates in flames., Appl. Opt. 31 (1992) 6547-57. doi:10.1364/AO.31.006547.

[14] A. Bescond, J. Yon, T. Girasole, C. Jouen, C. Rozé, A. Coppalle, Numerical investigation of the possibility to determine the primary particle size of fractal aggregates by measuring light depolarization, J. Quant. Spectrosc. Radiat. Transf. $126 \quad$ (2013) 130-139. doi:10.1016/j.jqsrt.2012.10.011.

[15]H.A. Michelsen, F. Liu, B.F. Kock, H. Bladh, A. Boiarciuc, M. Charwath, et al., Modeling laser-induced incandescence of soot: a summary and comparison of LII models, Appl. Phys. B. 87 (2007) 503-521. doi:10.1007/s00340-007-2619-5.

[16] M.L. Eggersdorfer, A.J. Gröhn, C.M. Sorensen, P.H. McMurry, S.E. Pratsinis, Massmobility characterization of flame-made $\mathrm{ZrO} 2$ aerosols: primary particle diameter and extent of aggregation., J. Colloid Interface Sci. 387 (2012) 12-23. doi:10.1016/j.jcis.2012.07.078.

[17] M.L. Eggersdorfer, D. Kadau, H.J. Herrmann, S.E. Pratsinis, Aggregate morphology evolution by sintering: number \& diameter of primary particles, J. Aerosol Sci. 46 (2012) 7- 
19. doi:10.1016/j.jaerosci.2011.11.005.

[18] R. Dastanpour, S.N. Rogak, B. Graves, J. Olfert, M.L. Eggersdorfer, A.M. Boies, Improved sizing of soot primary particles using mass-mobility measurements, Aerosol Sci. Technol. 50 (2015) 101-109. doi:10.1080/02786826.2015.1130796.

[19] A.M. Brasil, T.L. Farias, M.G. Carvalho, A recipe for image characterization of fractal-like aggregates, J. Aerosol Sci. 30 (1999) 1379-1389. doi:10.1016/S0021-8502(99)00026-9.

[20]R. Dastanpour, S.N. Rogak, Observations of a correlation between primary particle and aggregate size for soot particles, Aerosol Sci. Technol. 48 (2014) 1043-1049. doi:10.1080/02786826.2014.955565.

[21]C.K. Gaddam, R.L. Vander Wal, Physical and chemical characterization of SIDI engine $\begin{array}{lllll}\text { particulates, } & \text { Combust. } & \text { Flame. } & 160 & \text { (2013) }\end{array}$ doi:10.1016/j.combustflame.2013.05.025.

[22] Ü.Ö. Köylü, G.M. Faeth, T.L. Farias, M.G. Carvalho, Fractal and projected structure properties of soot aggregates, Combust. Flame. 100 (1995) 621-633. doi:10.1016/00102180(94)00147-K.

[23] A.I. Medalia, F.A. Heckman, Morphology of aggregates-II. Size and shape factors of carbon black aggregates from electron microscopy, Carbon N. Y. 7 (1969) 567-582. doi:10.1016/0008-6223(69)90029-3.

[24]P.J. De Temmerman, E. Verleysen, J. Lammertyn, J. Mast, Size measurement uncertainties of near-monodisperse, near-spherical nanoparticles using transmission electron microscopy and particle-tracking analysis, J. Nanoparticle Res. 16 (2014) 2628. doi:10.1007/s11051014-2628-3.

[25] T.J. Johnson, J.S. Olfert, J.P.R. Symonds, M. Johnson, T. Rindlisbacher, J.J. Swanson, et al., Effective Density and Mass-Mobility Exponent of Aircraft Turbine Particulate Matter, J. Propuls. Power. 31 (2015) 573-582. doi:10.2514/1.B35367.

[26] B. Graves, J. Olfert, B. Patychuk, R. Dastanpour, S. Rogak, Characterization of Particulate Matter Morphology and Volatility from a Compression-Ignition Natural-Gas DirectInjection Engine, Aerosol Sci. Technol. 49 (2015) 589-598. doi:10.1080/02786826.2015.1050482.

[27] A.M. Boies, M.E.J. Stettler, J.J. Swanson, T.J. Johnson, J.S. Olfert, M. Johnson, et al., Particle Emission Characteristics of a Gas Turbine with a Double Annular Combustor, Aerosol Sci. Technol. 49 (2015) 842-855. doi:10.1080/02786826.2015.1078452.

[28] K. Park, D.B. Kittelson, P.H. McMurry, Structural properties of diesel exhaust particles measured by transmission electron microscopy (TEM): relationships to particle mass and mobility, Aerosol Sci. Technol. 38 (2004) 881-889. doi:10.1080/027868290505189.

[29] I. Grishin, K. Thomson, F. Migliorini, J.J. Sloan, Application of the Hough transform for the automatic determination of soot aggregate morphology., Appl. Opt. 51 (2012) 610-20. doi:10.1364/AO.51.000610.

[30] A. Bescond, J. Yon, F.X. Ouf, D. Ferry, D. Delhaye, D. Gaffié, et al., Automated determination of aggregate primary particle size distribution by tem image analysis: application to soot., Aerosol Sci. Technol. 48 (2014) 831-841. doi:10.1080/02786826.2014.932896.

[31]P.J. De Temmerman, E. Verleysen, J. Lammertyn, J. Mast, Semi-automatic size 
measurement of primary particles in aggregated nanomaterials by transmission electron microscopy, Powder Technol. 261 (2014) 191-200. doi:10.1016/j.powtec.2014.04.040.

[32] T. Cleary, R. Samson, J.W. Gentry, Methodology for fractal analysis of combustion aerosols and particle clusters, Aerosol Sci. Technol. 12 (1990) 518-525. doi:10.1080/02786829008959366.

[33] N. Otsu, A threshold selection method from gray-level histograms, Automatica. 11(285-296 (1975) 23-27. http://web-ext.u-aizu.ac.jp/course/bmclass/documents/otsu1979.pdf (accessed December 17, 2015).

[34] M.L. Eggersdorfer, S.E. Pratsinis, Restructuring of aggregates and their primary particle size distribution during sintering, AIChE J. 59 (2013) 1118-1126. doi:10.1002/aic.14043.

[35] R. Botet, R. Jullien, M. Kolb, Hierarchical model for irreversible kinetic cluster formation, Phys. A Math. Gen. 17 (1984) 75-79. doi:10.1088/0305-4470/17/2/009.

[36] M.L. Eggersdorfer, S.E. Pratsinis, The structure of agglomerates consisting of polydisperse particles., Aerosol Sci. Technol. 46 (2012) 347-353. doi:10.1080/02786826.2011.631956.

[37]F.X. Ouf, J. Yon, P. Ausset, A. Coppalle, M. Maillé, Influence of Sampling and Storage Protocol on Fractal Morphology of Soot Studied by Transmission Electron Microscopy, Aerosol Sci. Technol. 44 (2010) 1005-1017. doi:10.1080/02786826.2010.507228.

[38] M. Wentzel, H. Gorzawski, K.-H. Naumann, H. Saathoff, S. Weinbruch, Transmission electron microscopical and aerosol dynamical characterization of soot aerosols, J. Aerosol Sci. 34 (2003) 1347-1370. doi:10.1016/S0021-8502(03)00360-4.

[39]D. Lottin, D. Ferry, J.-M. Gay, D. Delhaye, F.-X. Ouf, On methods determining the fractal dimension of combustion aerosols and particleclusters, J. Aerosol Sci. 58 (2013) 41-49. doi:10.1016/j.jaerosci.2012.12.009.

[40] P. Gwaze, O. Schmid, H.J. Annegarn, M.O. Andreae, J. Huth, G. Helas, Comparison of three methods of fractal analysis applied to soot aggregates from wood combustion, J. Aerosol Sci. 37 (2006) 820-838. doi:10.1016/j.jaerosci.2005.06.007.

[41]A. Soewono, Morphology and microstructure of diesel particulates, The University of British Columbia, 2008.

[42] A. Soewono, S. Rogak, Morphology and Microstructure of Engine-Emitted Particulates, SAE Tech. Pap. (2009). doi:10.4271/2009-01-1906.

[43] R. Dastanpour, S.N. Rogak, The effect of primary particle polydispersity on the morphology and mobility diameter of the fractal agglomerates in different flow regimes, J. Aerosol Sci. 94 (2016) 22-32. doi:10.1016/j.jaerosci.2015.12.005.

[44]R. Dastanpour, J. Boone, S.N. Rogak, PCM program, (2016). http://www.aerosol.mech.ubc.ca/research/ soot-and-nanoparticles/. 


\title{
Supplemental Information
}

\section{for \\ Automated Primary Particle Sizing of Nanoparticle Aggregates by TEM Image Analysis}

\author{
Ramin Dastanpour ${ }^{1, a}$, Jocelyne M. Boone ${ }^{1}$ and Steven N. Rogak ${ }^{1}$, \\ ${ }^{1}$ Department of Mechanical Engineering, University of British Columbia \\ 6250 Applied Sciences Lane, Vancouver, BC, Canada, V6T 1Z4
}

${ }^{a}$ Corresponding author: e-mail: r.dastanpour@alumni.ubc.ca; Fax: +1-604-822-2403 
PCM error when average $\mathrm{P}^{*}$ obtained from the simulation of the fractal agglomerates is used for straight chains:

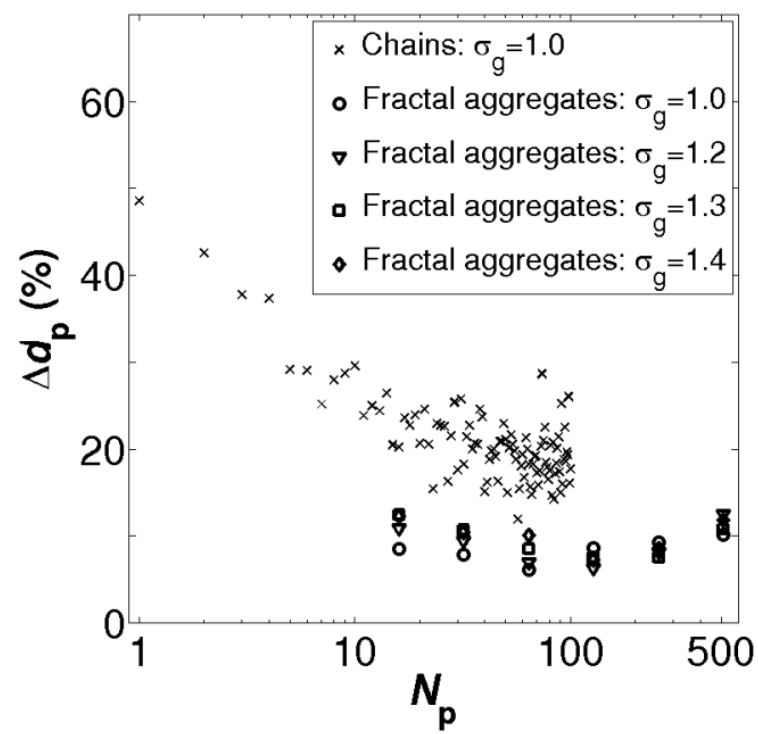

Figure SI1: Primary particle sizing error of PCM for chains and aggregates of different sizes and polydispersity. Chain results are governed with the application of $\mathrm{P}^{*}=0.84$ (corresponding to fractal agglomerates of monodisperse monomers $\sigma_{g}=1$ )

Comparison of the manually measured average diameters of the primary particles in individual aggregates with $\mathrm{PCM}$, when average $\mathrm{P}^{*}$ obtained from the simulation of the fractal agglomerates is used for the analysis of the TEM images:

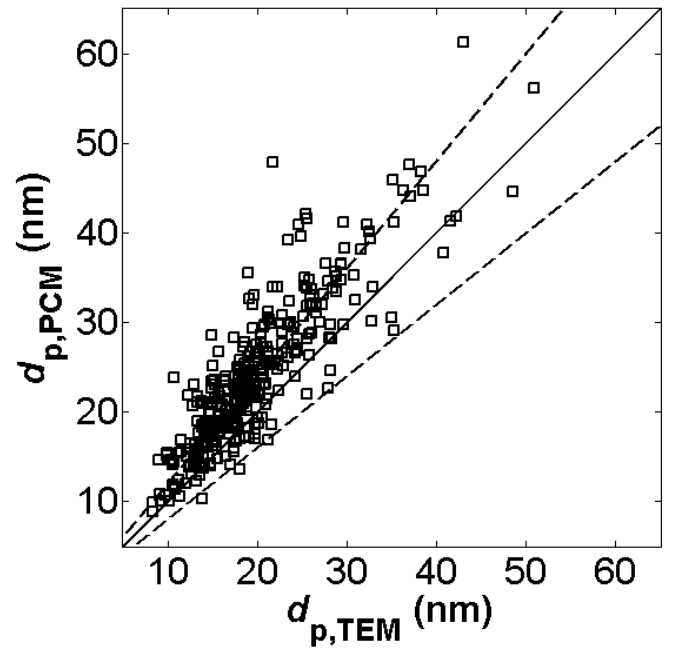

Figure SI2: Average diameter of the primary particles estimated by $\mathrm{PCM}$ (using $\mathrm{P}^{*}=0.85$, obtained for point-touching agglomerates with $\sigma_{g}=1.2$ ) vs. TEM (manual) sizing. Solid line is a 1-by-1 line (0\% error), and dashed lines are $\pm 20 \%$ error boundaries. Figure consists of 390 data points. 


\section{Generalized model}

An attempt was made to develop a general method for particles of all shapes and structures. To this end, the variation of $\mathrm{P}^{*}$ with four sets of parameters was investigated (Figure SI3): i) aspect ratio of the projected particles $(L / W)$, where $L$ and $W$ are the maximum length and width of the projected particle, respectively; ii) slope of the pair correlation function at two-dimensional gyration radius, $d \mathrm{P}\left(R_{\mathrm{g}}\right) / d r$; iii) ratio of $d_{\mathrm{a}} / d_{\mathrm{g}}$; iv) ratio of $d_{\mathrm{a}} / L_{\text {skel }}$ where $L_{\text {skel }}$ is the length of the particle skeleton. The first three parameters are correlated to the fractal structure of the particles while the last one is a measure of the average particle thickness. As shown in Figures SI3 and SI4, a simple correlation between the parameters described above and the value of the $\mathrm{P}^{*}$ cannot be easily derived. The following more complex two-parameter regression was obtained for $\mathrm{P}^{*}$ as a function of $d \mathrm{P}\left(R_{\mathrm{g}}\right) / d r$ and $L / W\left(\mathrm{R}^{2}=0.94\right)$ :

$\mathrm{P}_{\text {gen }}^{*}=\left(\frac{\mathrm{P}_{\mathrm{avg}}^{*}\left(\sigma_{\mathrm{g}}\right)}{0.84}\right)\left[0.7+0.003\left(\left.\frac{d \mathrm{P}}{d r}\right|_{R_{\mathrm{g}}}\right)^{-0.24}+0.2\left(\frac{L}{W}\right)^{-1.13}\right]$

where $\mathrm{P}_{\mathrm{avg}}^{*}$ should be calculated from Eq. (2) for point-touching agglomerates and is equal to 0.913 for real soot aggregates.
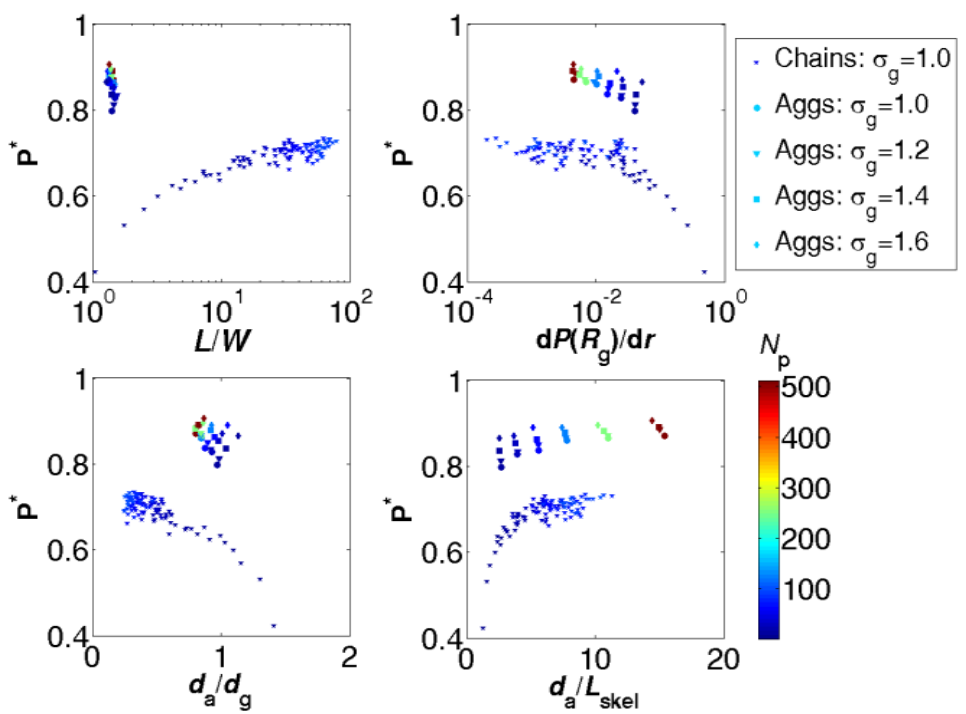

Figure SI3: Variation of $\mathrm{P}^{*}$ with i) aspect ratio $(L / W)$ where $L$ and $W$ are the maximum length and width of the projected particle, respectively; ii) slope of the pair correlation function at two-dimensional gyration radius, $\mathrm{dP}\left(R_{\mathrm{g}}\right) / \mathrm{dr}$; iii) ratio of $d_{\mathrm{a}} / d_{\mathrm{g}}$; iv) ratio of $d_{\mathrm{a}} / L_{\text {skel }}$ where $L_{\text {skel }}$ is the length of the particle skeleton. 


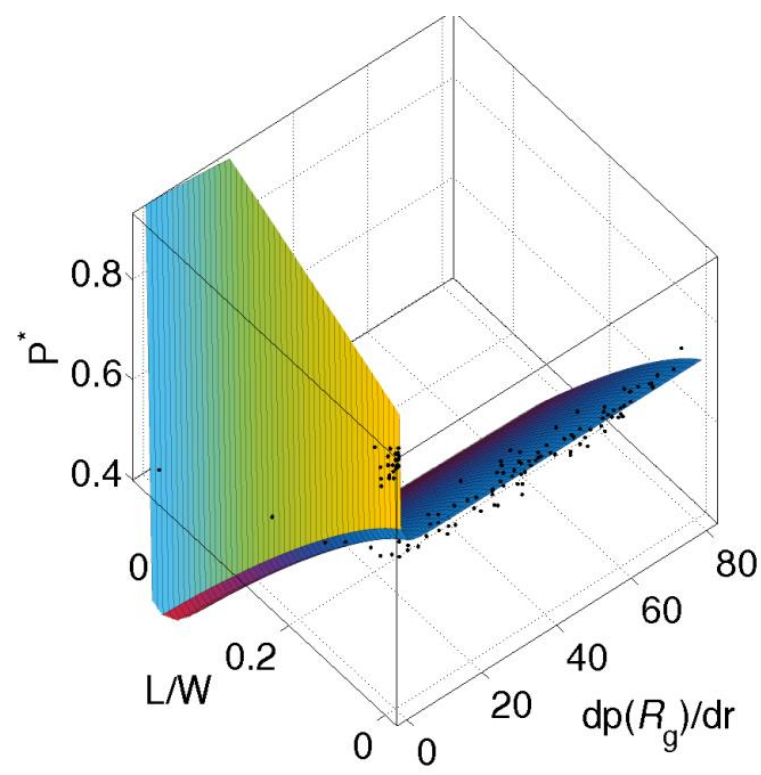

Figure SI4: Variation of $\mathrm{P}^{*} \mathrm{~L} / \mathrm{W}$ and $\mathrm{dP}\left(\mathrm{R}_{\mathrm{g}}\right) / \mathrm{dr}$; and corresponding surface fit.

As shown in Figure SI6, while the generalized model has the advantage of using a single correlation for all particles, its accuracy is slightly lower than the simple PCM with a value of $\mathrm{P}^{*}$ optimized for a particular class of particle. Using the generalized model for the analysis of the TEM images, ensemble-average diameter of the primary particles and average $d_{\mathrm{p}}$ for individual aggregates can be calculated with approximately $5 \%$ and $15 \%$ error, respectively.
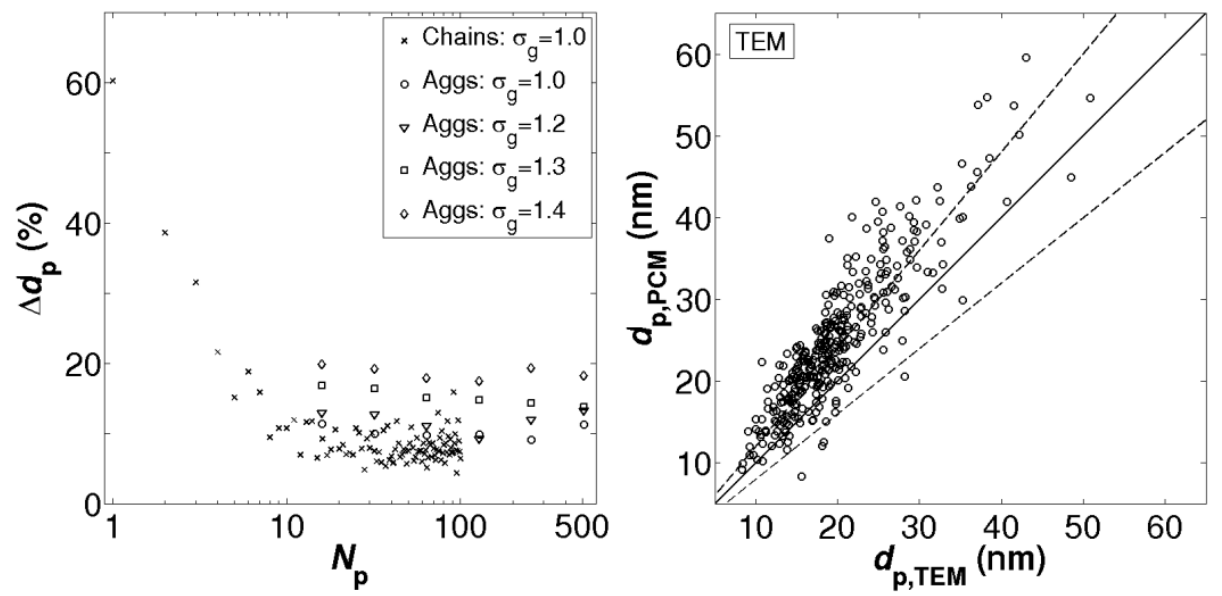

Figure SI5: Generalized model error for chains and agglomerates composed of point-touching monomers, and real TEM images of carbon soot. Solid line is a 1-by-1 line ( $0 \%$ error), and dashed lines are $\pm 20 \%$ error boundaries.

The generalized version of the PCM is advantageous when the structures of the particles under investigation and substantially different (e.g. when the sample contains both chains and fractal clusters to the approximately same extent); however, for most collections of the images, particle structures and not enormously different and simple PCM is preferable. 
When either versions of the PCM are used, errors associated with the human biases and manual analysis of the TEM images are eliminated. As illustrated in Figure SI6, both versions of this method are capable of capturing a size correlation between the primary particle and aggregate sizes, consistent with the trends reported by Dastanpour and Rogak (2014) from the manual analysis.
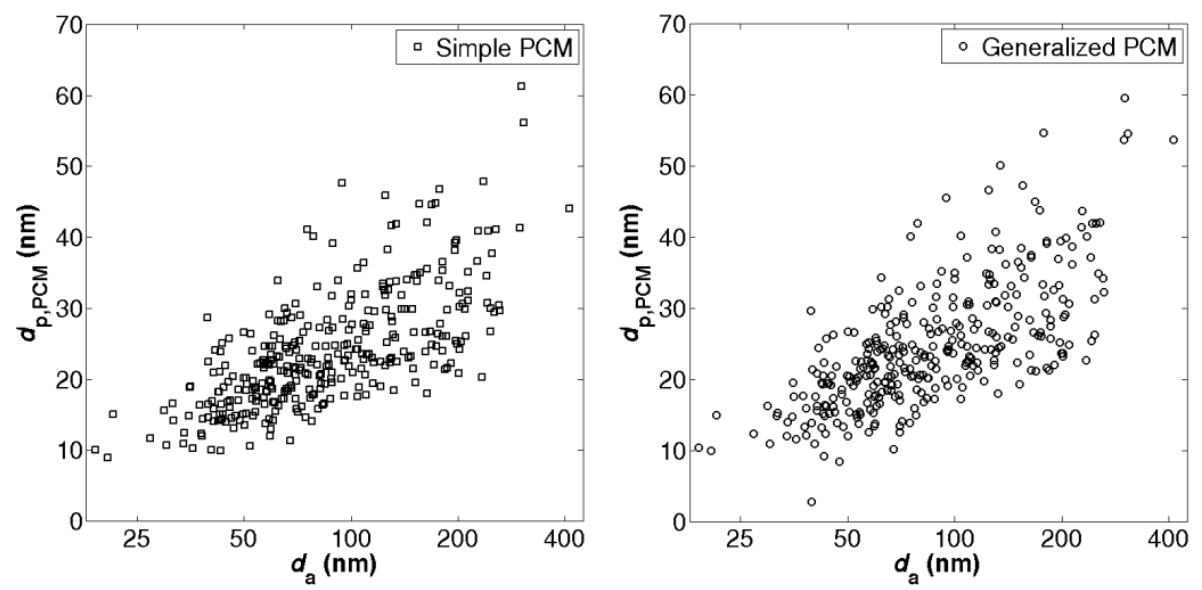

Figure SI6: Variation of average primary particle diameter (in individual aggregates) vs. aggregateprojected area equivalent diameter. Diameters measured by PCM. 